\title{
A First Analysis of a New Fixed Point Iteration of the Boltzmann Equation: Application to TCAD
}

\author{
Vincent Peikert \\ Swiss Federal Institute of Technology \\ Zurich, Switzerland \\ Email: vpeikert@iis.ee.ethz.ch
}

\author{
Andreas Schenk \\ Swiss Federal Institute of Technology \\ Zurich, Switzerland \\ Email: schenk@iis.ee.ethz.ch
}

\begin{abstract}
This paper presents a first analysis of a new general fixed point iteration of the Boltzmann transport equation. The scheme is based on a recent theory on Inverse Scattering Operators. Due to the fact that the implementation of this scheme is extremely involved, the expansion is truncated after the second iteration as a start. Comparisons with Monte Carlo simulations verify that the second iteration step gives sufficient corrections to the equilibrium distribution in bulk silicon, if the external field is not too large. However, it turns out that the second iteration is not sufficient to address inhomogeneous semiconductors. One reason is that a term containing the built-in electric field is not compensated in this order. Moreover, even in regions with low electric field and with small gradients of the quasi-Fermi level the second-order solution deviates notably from Monte Carlo simulations. Although this scheme has a lot of potential for TCAD applications, the adaptability is not straight-forward and further analysis of higher order terms is necessary.
\end{abstract}

\section{The Boltzmann Transport Equation}

The stationary Boltzmann transport equation (BTE) describes the single particle probability density $f(\vec{r}, \vec{k})$ in semiconductor devices given an electric field $\vec{E}$ :

$$
\vec{v}(\vec{k}) \nabla_{r} f(\vec{r}, \vec{k})-\frac{q}{\hbar} \vec{E} \nabla_{k} f(\vec{r}, \vec{k})=S f(\vec{r}, \vec{k})
$$

where $q$ is the particle charge, $\vec{v}(\vec{k})$ is the group velocity of the particle and $S$ is the scattering operator which describes the change of $f$ in time due to scattering processes. The BTE can be derived from Schrödinger's equation under approximations that are still valid in state-of-the-art semiconductor devices.

The current density is given by

$$
\vec{j}(\vec{r})=\frac{q}{(2 \pi)^{3}} \int_{V_{B z}} d^{3} k \vec{v}(\vec{k}) f(\vec{r}, \vec{k})
$$

meaning that the knowledge of $f$ is necessary to design semiconductor nano-devices. An accurate numerical solver as part of a TCAD environment could lower costs and speed up the engineering process significantly.

However, the direct numerical solution of the BTE is still an open issue due to the so-called curse of dimensionality: Since the probability density $f(\vec{r}, \vec{k})$ is a function in a six-dimensional phase space, a full tensor grid would scale with $2^{N \times 6}$ where $2^{N}$ indicates the number of discretization points per dimension. For that reason Monte Carlo methods are frequently used to extract an empirical distribution function by simulating independent paths of particles (this method converges with $1 / \sqrt{N}$ ). The main problem of the Monte Carlo approach is its high computational cost. Additionally, Monte Carlo is not able to simulate very rare events adequately although some rare scattering processes can have a large impact on the current (for example in floating body devices).

Another approach to derive the current density is the introduction of so-called moment equations which are equations for moments of $f$ over the momentum space only. Hence, these equations are not $\vec{k}$-dependent anymore and still form the standard for commercial TCAD tools (cf. [1]). The most important example is the drift-diffusion equation:

$$
\vec{j}=q \mu n \vec{E}+q D \nabla_{r} n .
$$

The drawback of moment equations is that they contain transport parameters (e.g. diffusivity $D$ and mobility $\mu$ in Eq. (3)) which require additional models themselves. Finding proper models for transport parameters is still an open problem, since all common models are known to fail significantly if the systems are far away from equilibrium.

This paper investigates new exact expressions for DriftDiffusion transport parameters. With these exact expressions the same current density $j$ results from Eq. (3) compared to solving Eq. (1) and then computing $\vec{j}$ via Eq. (2).

\section{Exact Drift-Diffusion Transport Parameters}

For semiconductor devices it is assumed that particles propagate in the mean field of all other particles of the same type and that there is no other interaction between them. This means that the scattering operator $S$ becomes linear and can be inverted numerically as it was first shown in [2]. However, because $S$ has a kernel defining the equilibrium distribution function $f_{e q}$, only the non-equilibrium part of $f$ reading $f-f_{e q}$ can be inverted. Hence, under the application of the Inverse Scattering Operator $S^{-1}$ (ISO) Eq. (1) can be written as 


$$
S^{-1} \vec{v} \nabla_{r} f-S^{-1} \frac{q}{\hbar} \vec{E} \cdot \nabla_{k} f=f-\frac{n}{n_{e q}} f_{e q} .
$$

On the right-hand side of Eq. (4), the distribution function $f(\vec{r}, \vec{k})$ turns out isolated when bringing $\frac{n}{n_{e q}} f_{e q}$ to the lefthand side. Multiplying by $\vec{v}(\vec{k})$ and integrating over $\vec{k}$ gives an exact moment equation for the current density $\vec{j}(\vec{r})$ (cf. Eq. (2)) which can be directly transformed into the form in Eq. (3). The so defined exact drift-diffusion transport parameters read (see [2])

$$
\begin{aligned}
\mu_{n m} & =-\frac{q}{n(\vec{r}) \hbar} \int_{B z}\left(\nabla_{k}\right)_{m} S_{v_{n}}^{-1}(\vec{k}, b) f(\vec{k}, b) d^{3} k \\
D_{n m} & =-\frac{1}{n(\vec{r})} \int_{B z} S_{v_{n}}^{-1}(\vec{k}, b) v_{m}(\vec{k}, b) f(\vec{k}, b) d^{3} k
\end{aligned}
$$

where $S_{g}^{-1}\left(\vec{k}_{1}, \vec{r}\right):=\int g(\vec{k}) S^{-1}\left(\vec{k}, \vec{k}_{1}, \vec{r}\right) d^{3} k$.

As described in [3], these tensorial transport parameters can be obtained by replacing $f$ with a Monte Carlo simulated empirical distribution function. Another ansatz to derive $f$ is to consider Eq. (4) as a fixed point equation for $f$ and to implement the resulting expressions for $\mu$ and $D$ up to a sufficient order. This new iterative scheme was first proposed in [4] and builds - to the authors best knowledge - the only known exact parametrization of drift-diffusion transport parameters.

Using the property $S^{-1}\left|f_{e q} h>=f_{e q}\right| S_{h}^{-1}>$ (see [5] p. 250) and with $f=\frac{n}{n_{e q}} f_{e q}(1+H)$, the iterative scheme (4) turns into

$$
H=-\beta \nabla_{r} \psi S_{\vec{v}}^{-1}-\beta \nabla_{r} \psi S_{\vec{v} H}^{-1}+\frac{q}{\hbar} \nabla_{r} \phi S_{\nabla_{k} H}^{-1}+S_{\vec{v} \nabla_{r} H}^{-1} .
$$

It is worth to point out that the distribution function (and therefore the transport parameters) does not only depend on the two driving forces 'gradient of the electrostatic potential' $\nabla \phi$ and 'gradient of the quasi-Fermi potential' $\nabla \psi$, as assumed in standard models, but also on higherorder derivatives of these potentials. Depending on the convergence properties of fixed point (6) and considering possible applications for TCAD, three cases are thinkable: In the best case this scheme could form a new direct method to solve the BTE equation. This method would neither need a long simulation time nor a high memory consumption. The second best application for this scheme would be a coupling with the new Monte Carlo method proposed in [4]: in regions where an implemented order is not sufficient, the empirical distribution function can be applied. The worst case would be that the scheme converges extremely slowly and uncontrollably such that the implementation is not reasonable or that the scheme does not converge at all.
This paper is a first progress report on the convergence issue and points out the problematic regions for the second iteration approximation.

\section{DISCUSSION}

In the following, $f_{e q}$ is assumed to be a MaxwellBoltzmann-distribution

$$
f_{e q}=\underbrace{e^{-\frac{\epsilon(\vec{k})}{k T}}}_{h_{e q}(\vec{r})} \underbrace{e^{\frac{q \phi_{e q}(\vec{r})-q \psi_{e q}}{k T}}}_{\frac{n_{e q}(\vec{r})}{N_{C}}}
$$

meaning that Pauli's principle is neglected. This narrowing is valid in many devices (doping is smaller than $10^{19} \mathrm{~cm}^{-3}$ ). In non-equilibrium the density $n$ can be expressed by the wellknown transformation $n=N_{C} e^{\frac{q \phi(\vec{r})-q \psi(\vec{r})}{k T}}$.

\section{A. The Fixed Point With Simplest Scattering Model}

To get a first idea of the convergence properties of Eq. (6) the simplest scattering case is considered when $S f(\vec{k}, \vec{r})$ can be expressed by a relaxation time $\left(\frac{\partial f}{\partial t}\right)_{\text {Scattering }}=\frac{f-f_{e q}}{\tau}$. Eq. (4) can then be rewritten as

$$
\underbrace{\tau \vec{v} \nabla_{r} f}_{T_{1}}-\underbrace{\tau \frac{q}{\hbar} \vec{E} \cdot \nabla_{k} f}_{T_{2}}+\frac{n}{n_{e q}} f_{e q}=f .
$$

The mth order is given by:

$$
f_{m}(\vec{r}, \vec{k})=\sum_{i=0}^{m} \underbrace{\left(T_{1}-T_{2}\right)^{i} n h_{e q}}_{\hat{f}_{i}}
$$

and the first two iterations read $f_{0}=n h_{e q}$ and

$$
\begin{array}{r}
f_{1}=\left(\tau \vec{v}\left(\frac{-q \vec{E}(\vec{r})-q \nabla_{r} \psi(\vec{r})}{k T}\right)+\tau q \frac{\vec{v}}{k T} \vec{E}+1\right) n h_{e q} \\
=\left(-\tau q \vec{v} \frac{\nabla_{r} \psi(\vec{r})}{k T}+1\right) n h_{e q} .
\end{array}
$$

The first order is independent of $\vec{E}$ so that possible built-in fields do not lead to strong corrections in the first order.

The convergence for $\hat{f}_{i}$ if $i \rightarrow \infty$ has to be explored. However the problem is that the operators $T_{1}$ and $T_{2}$ do not commute $\left(\left[T_{1}, T_{2}\right]=T_{1} T_{2}-T_{2} T_{1} \neq 0\right)$ so that all mixing terms have to be considered. This leads to complex combinatoric problems if trying to explicitly express $\hat{f}_{i}$. For linear $\psi$, linear $\phi$ and constant $\tau$ as well as linear band structure, $\epsilon(\vec{k})=\vec{v} \vec{k}$ (as for example approximately given in graphene) $\left[T_{1}, T_{2}\right]=0$ and $\hat{f}_{i}$ is given by

$$
\hat{f}_{i}=(-1)^{i}\left(\tau \vec{v} q \frac{\nabla_{r} \psi}{k T}\right)^{i} n h_{e q} .
$$

If and only if $\left|\tau \vec{v} q \frac{\nabla_{r} \psi}{k T}\right|<1$, the series (9) converges to

$$
\sum_{i=0}^{\infty} \hat{f}_{i}=\frac{1}{1+\tau \vec{v} q \frac{\nabla_{r} \psi}{k T}} n h_{e q} .
$$




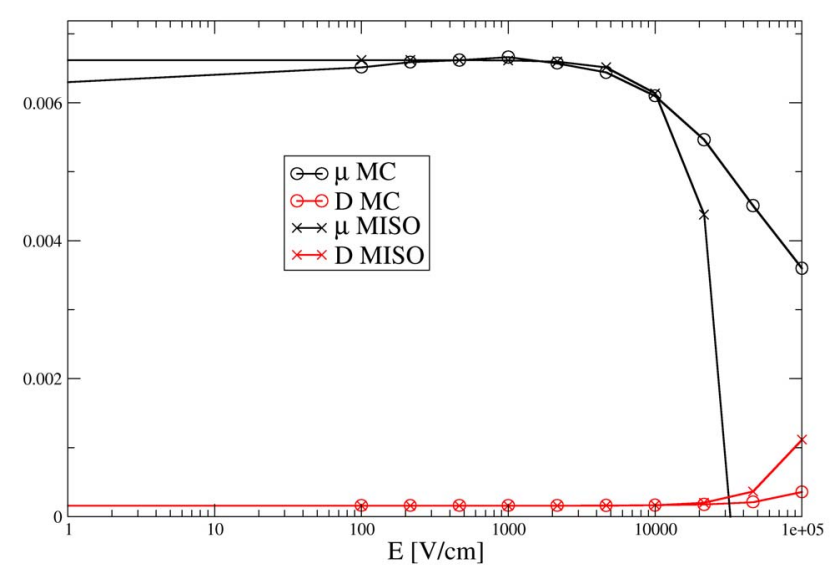

Fig. 1. Bulk transport parameters: comparison between MC and Eq. (13) using doping concentration of $10^{20} \mathrm{~cm}^{-3}$ arsenic doping.

Already in this simple case one can see that the fixed point only converges if the scattering rate is large compared to the external fields and that the speed of convergence is ruled by these parameters. Further if any of the external parameter functions is not linear or constant, then the electric field does not cancel out exactly anymore. That means that the convergence will depend on the strength of the built-in fields and not only on the applied external voltage.

\section{B. Second iteration step}

The second iteration step $H_{2}$ of the general scheme (6), starting with $H_{0}=0$ and $H_{1}=-\beta \sum_{i}^{3} \nabla_{r, i} \psi S_{\vec{v}_{i}}^{-1}$ reads:

$$
\begin{aligned}
& H_{2}=\sum_{i, j}^{3}(\underbrace{-\beta \nabla_{r, i} \psi S_{\vec{v}_{i}}^{-1}}_{\text {1th Order }}+\underbrace{\beta^{2} \nabla_{r, i} \psi \nabla_{r, j} \psi S_{\vec{v}_{j} S_{\vec{v}_{i}}^{-1}}^{-1}}_{\text {2nd Order I }} \\
& -\underbrace{\beta \frac{q}{\hbar} \nabla_{r, i} \psi \nabla_{r, j} \phi S_{\nabla_{k, j} S_{\vec{v}_{i}}^{-1}}^{-1}}_{\text {2nd Order II }}-\underbrace{\beta \nabla_{r, j}\left(\nabla_{r, i} \psi\right) S_{\vec{v}_{j} S_{\vec{v}_{i}}^{-1}}^{-1}}_{\text {2nd Order III }} \\
& -\underbrace{\beta \nabla_{r, i} \psi S_{\vec{v}_{j} \nabla_{r, j} S_{\vec{v}_{i}}^{-1}}^{-1}}_{\text {2nd Order IV }}) \text {. }
\end{aligned}
$$

\section{Bulk Semiconductor}

In bulk, $\nabla_{r} \psi=\nabla_{r} \phi, \nabla_{r}^{2} \psi=0$, and $\nabla_{r} S_{\vec{v}}^{-1}=0$ hold, hence only a second-order polynomial in $\nabla_{r} \phi$ is left from Eq. (13), if a homogeneous electric field is applied. The comparison with Monte Carlo simulations in Figure 1 show the expected behavior. The second iteration is sufficient, if the external field is not too large meaning that the homogeneous system is close to its equilibrium.

\section{Inhomogeneous Semiconductor}

In inhomogeneously doped semiconductors strong electric fields can exist even in equilibrium. They are caused by the thermal motion of charge carriers from highly doped to lowly doped regions (diffusion). Therefore, the relevant driving force is less the electric field than the gradient of the quasi-Fermi potential $\nabla \psi$ which is zero in equilibrium.

All corrections of scheme (6) contain a gradient of the quasi-Fermi potential so that they turn to zero in equilibrium. However, as soon as a small external voltage is applied, term II in Eq. (13) can become arbitrarily large. This term is not compensated in the second order, hence Eq. (13) could fail in regions with a built-in potential.

Nevertheless, where the gradient of the quasi-Fermi potential dominates (being still small since the applied voltage is also small) the second order (13) could be a reasonable approximation. The latter situation is given in the middle of the simulated $\mathrm{N}^{+} \mathrm{NN}^{+}$device (see Figure 2), where a low voltage (here $0.01 \mathrm{~V}$ ) is applied. However, it turns out that the corrections are too large compared to Monte Carlo simulations (see Figure 3). Obviously, the second iteration is not sufficient in inhomogeneous semiconductors even if the system is close to equilibrium. It is worth noting that at least the large gradient of the mobility in the middle of the $\mathrm{N}^{+} \mathrm{NN}^{+}$device is caused by the built-in field and not by the gradient of the quasi-Fermi potential (see left part of Figure 4). On the other hand, the large gradient of the diffusivity is caused by the second derivative of the gradient of the quasi-Fermi potential (see right part of Figure 4).

\section{CONCLUSION}

The second iteration of the new fixed point of the BTE is not sufficient to describe the physics in semiconductor regions with strong built-in electric fields or (more general) large differences between gradient of the quasi-Fermi potential and electric field. Therefore, further analysis is essential. This discussion is related to the long-standing issue of the physics within built-in electric fields (see for example [6]) and could shed new light on it. As a next step, the general scheme (6) has to be studied in simple cases where the external parameter functions are polynomials.

\section{REFERENCES}

[1] SYNOPSIS INC. Sentaurus Device User Guide, Version Z-2007.03

[2] S. C. Brugger, A. Schenk, W. Fichtner, Moments of the Inverse Scattering Operator of the Boltzmann Equation: Theory an Applications, SIAM J. APPL. MATH. 2006.

[3] S. C. Brugger and A. Schenk, New One-Particle Monte Carlo Method for Nanoscale Device Simulation, Proc. 9th annual NSTI Nanotechnology Conference and Trade Show, Boston, Massachusetts, 2006.

[4] S.C. Brugger, V. Peikert, A. Schenk, Coupling the Monte-Carlo Method with Semi-Analytical Solutions of the Boltzmann Transport Equation, SISPAD, Hakone, Japan, 2008.

[5] S. C. Brugger, Computation of semiconductor properties using moments of the inverse scattering operator of the Boltzmann equation, Ph.D. dissertation, ETH Zurich, published at Hartung Gorre Verlag, Konstanz, 2005. [Online]. Available: http://ecollection.ethbib.ethz.ch

[6] C. Jungemann, T. Grasser and B. Meinerzhagen Failure of MomentsBased Transport Models in Nanoscale Devices Near Equillibrium, IEEE Transaction On Electron Devices, VOL. 52, 2005. 

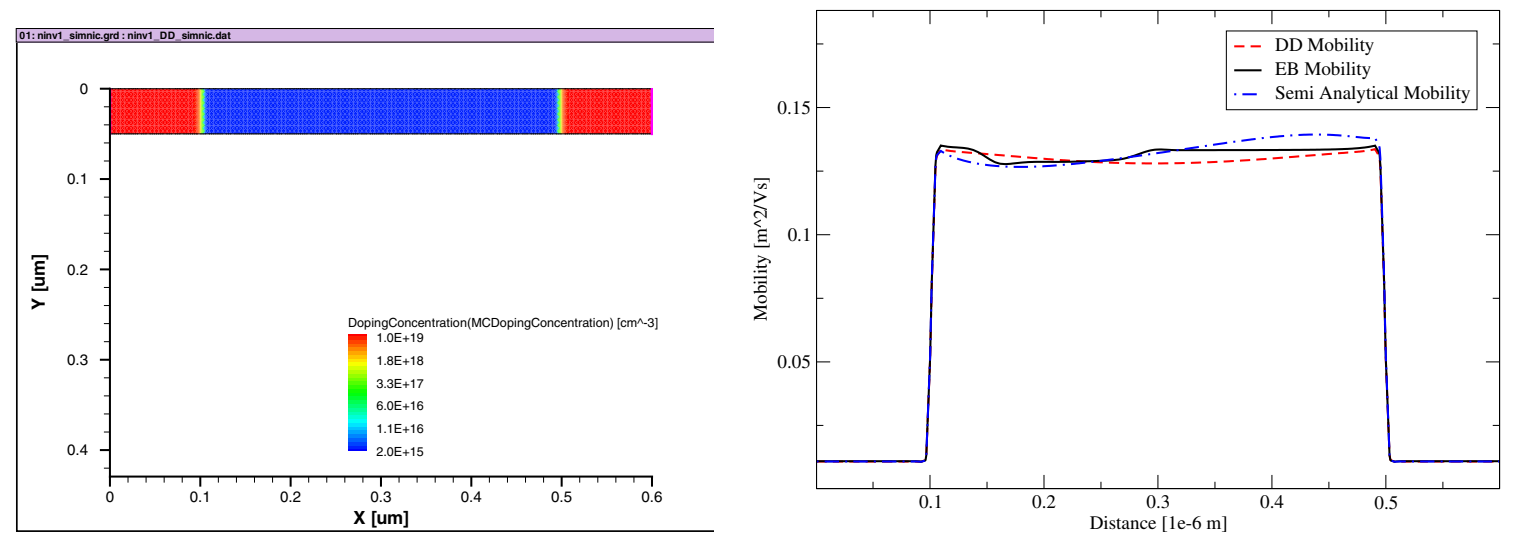

Fig. 2. Left: Simulated $\mathrm{N}^{+} \mathrm{NN}^{+}$device. Right: Standard drift-diffusion and energy-balance mobilities compared with Eq. (5) using Eq. (13).
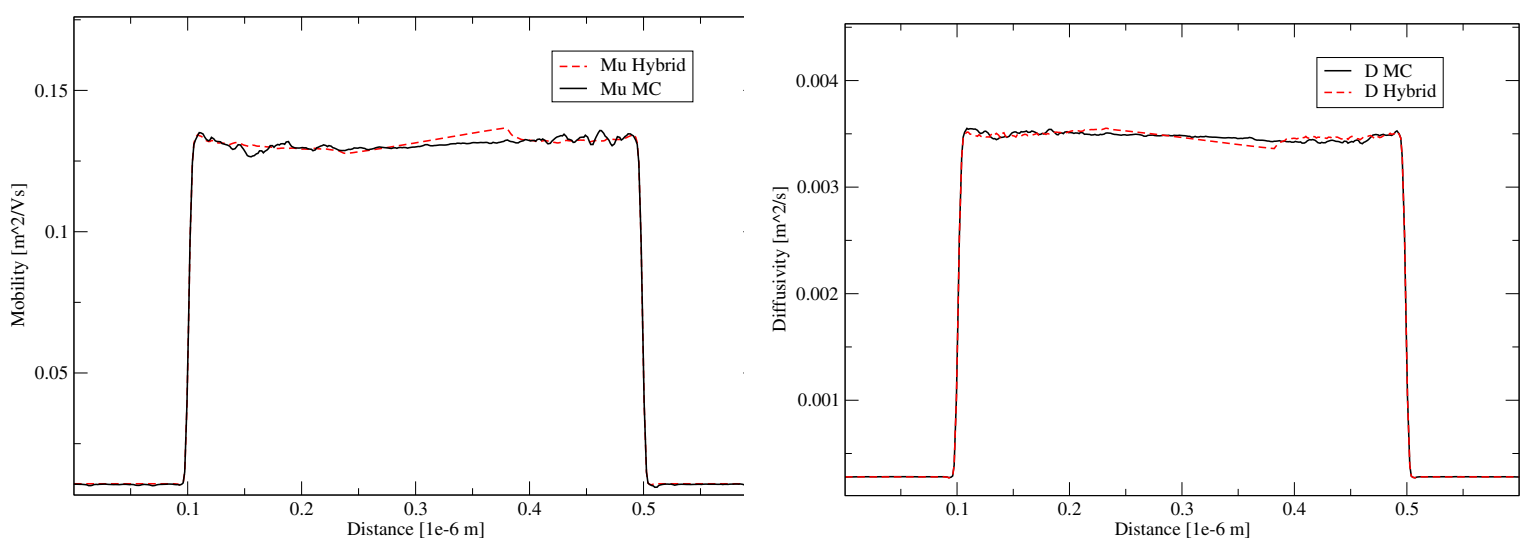

Fig. 3. The transport parameters in Eq. (5) derived with Eq. (13) in comparison to Monte Carlo. The transport parameters close to the $\mathrm{N}^{+} \mathrm{N}$ and $\mathrm{NN}^{+}$ junctions are replaced by other models.
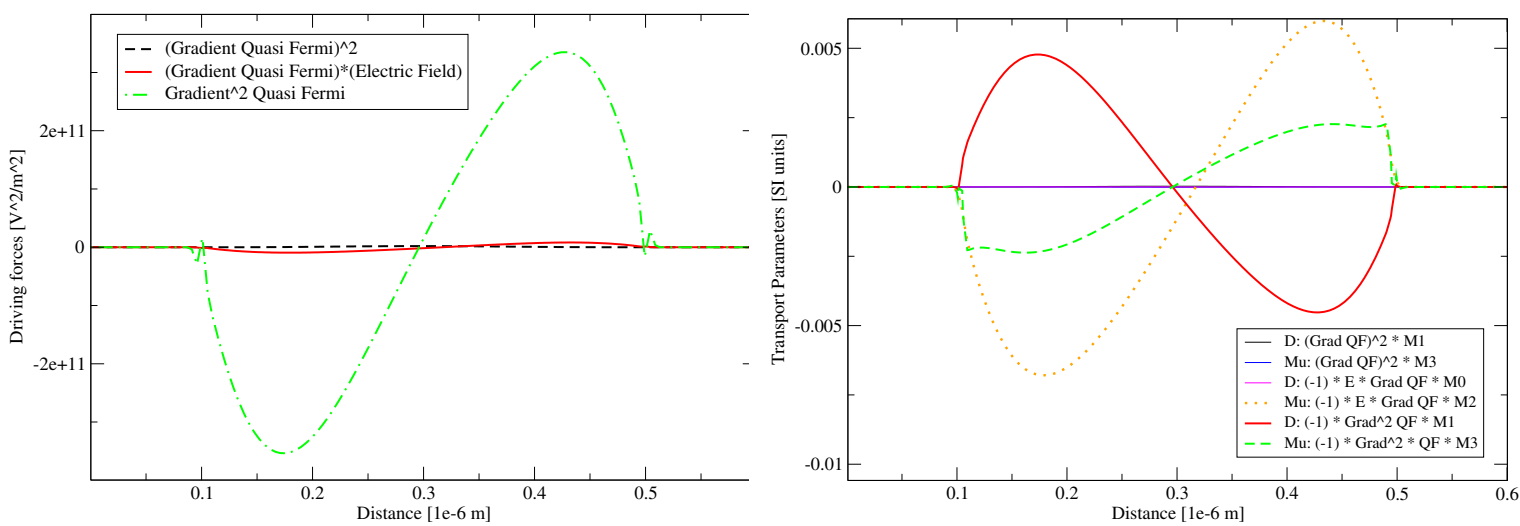

Fig. 4. Left: Forces in Eq. (13). Right: Corrections to the equilibrium transport parameters by Eq. (13). 